\title{
Neoadjuvant chemotherapy followed by radiotherapy versus radiotherapy alone in locally advanced carcinoma cervix: a prospective randomized study.
}

\author{
Dr Vikas Fotedar ${ }^{1}$, Dr Rajeev K Seam ${ }^{2}$, Dr Manoj K Gupta ${ }^{3}$, Dr Anupam \\ Jhobta ${ }^{4}$, \\ ${ }^{I} M D$ Radiotherapy, Senior Resident, Deptt. of Radiotherapy, IGMC, Shimla. \\ ${ }^{2} M D$ Radiotherapy, Prof \& HOD, Deptt. of Radiotherapy, IGMC, Shimla. \\ ${ }^{3}$ MD Radiotherapy, Prof, Deptt. of Radiotherapy, IGMC, Shimla. \\ ${ }^{4}$ MD Radiodiagnosis Asstt. Prof., Deptt of Radiodiagnosis, IGMC, Shimla.
}

\begin{abstract}
Study Objective: To compare the disease response, disease free survival,overall survival and toxicity profile to neoadjuvant chemotherapy followed by radiotherapy(CT-RT group) versus radiotherapy alone(RT alone) in locally advanced carcinoma cervix.

Materials and Method:Between July 2007 and August 2008, 113 patients with squamous cell carcinoma, adenocarcinoma and adenosquamous carcinoma cervix, FIGO stage IIB-IIIB, were randomized to receive either two cycles of cisplatin and 5-flourouracil(CT) followed by radiotherapy(RT) (CT-RT group $, n=58)$ or $R T$ alone ( $R T$ alone group , $n=55$ ).

Result \& Conclusion: In the CT-RT group 54 evaluable patients 52 responded: clinical complete response (cCR) in 1(1.85\%) and partial response in 51(94.45\%). Remaining 2 patients (3.70\%) had progressive disease (PR). Of 52 patients completed RT as planned. Following RT 38(73.08\%) achieved clinical CR, 13(25\%) had residual disease, and 1(1.92\%) had progressed at first follow up after 1 month. The stage of disease, histological grade, had no influence on the response to CT. Nausea /vomiting were the major side effects of CT. In the RT group, 54 patients were evaluable 41(75.93\%) patients achieved clinical CR, $12(22.22 \%)$ had residual disease, and 1(1.85\%) progressed. The side effects of RT were lower GI toxicity (diarrhea) and local skin reaction. These were equally distributed between the two groups. After 36 months of follow up, $c$ CR was seen in 34(64.15\%) patients, PR in 8(15.09\%) patients and 11(20.76\%) patients had died in CT-RT group. In RT alone group 36(66.67\%) patients had cCR, 8(14.82\%) patients had PD and 10 (18.51\%) patients had died. There were no significant difference in overall and disease free survival in the two groups.

Key words: Carcinoma cervix, Locally advanced,Neoadjuvant chemotherapy, Radiotherapy and Survival.
\end{abstract}

\section{Introduction:}

Cervical carcinoma is the second most frequent cancer among women worldwide and most frequent cancers among women in Africa, South America and Asia including India ${ }^{[1]}$.Squamous cell carcinoma is the most common cancer among Indian women. It accounts for 20-43 cases per 100000 population ${ }^{[2]}$. The majority (approx 80\%) present with locally advanced disease ${ }^{[3]}$ (FIGO IIB- IVA) due to lack of screening The five year survival rate with radiotherapy (RT) is about $60 \%$ in stage IIB, 30-35\% in Stage IIIB, and less than $15 \%$ in stage IVA disease. Failure is accounted by local recurrence in $40-70 \%$ and distant failure in about $20-25 \%$ patients $^{[4]}$. The large volume of primary tumor and presence of micrometastatic disease at diagnosis are important reasons for RT treatment failure. 'Treatment of the recurrent disease is mainly palliative by chemotherapy (CT). The response rate averages $25-60 \%$, median duration of response is 4-6 months and survival duration is 6-10 months. The concurrent chemoradiotherapy (CRT) is the standard of care for locally advanced cervical carcinoma at present ${ }^{[5]}$. In 1999, the National Cancer Institute ${ }^{[6]}$ released a clinical announcement citing the results of the five positive trials, and recommending that "strong consideration should be given to adding chemotherapy to radiation therapy in the treatment of invasive cervical cancer. Following this NCI alert CRT became the standard of care which was again proven to have benefit in subsequent meta-analyses ${ }^{[7]}$

Two such randomized controlled trials were conducted in $2004 \& 2005$ in our centre which did not show any benefit over RT alone and at the same time had higher toxicity ${ }^{[8]}$ So the present study was conducted with the following aim and objectives:

To compare the disease response, local control,disease free survival and overall survival to neoadjuvant chemotherapy followed by radiotherapy and radiotherapy alone in locally advanced carcinoma cervix.

To compare toxicity profile following administration of neoadjuvant chemotherapy followed by radiotherapy and radiotherapy alone in locally advanced carcinoma cervix. 
To compare the patients suitability for brachytherapy following administration of chemotherapy followed by radiotherapy and radiotherapy alone in locally advanced carcinoma cervix.

\section{Materials And Method}

Prospective randomized study was conducted for a period of one year from Sept. 07 to Aug 08 in 113 patients, in the Department of Radiotherapy \& Oncology, Regional Cancer Centre, IGMC, Shimla; in patient with histological proven invasive squamous cell carcinoma, adenocacinoma and adenosquamous carcinoma. Ethical approval to conduct the study was obtained from the Institutional Review Board of Indira Gandhi Medical College and Hospital, Shimla(HP) India. Written consent was obtained from the subjects.

The subjects with locally advanced cervical cancer (FIGO IIB- IIIB) were randomized to receive two cycles of Cisplatin plus 5- Flurouracil (5-FU) CT followed by RT (CT-RT group, n= 58) or RT alone (RT group, $\mathrm{n}=55$ ). Patients were registered in a gynaecological tumor clinic and were examined by two Radiation Oncologists. Eligibility criteria included previously untreated patients, biopsy proven squamous cell carcinoma/ adenocarcinoma/adeno squamous, FIGO stage IIB-IIIB, Karnofsky status $>50$, and no significant hematological, hepatic or renal impairment as judged by standard hematological and biochemical investigations. Eligible subjects were randomized in two groups by a lot method by a third person not involved with the study.Baseline blood investigation, Chest X-ray and ultrasound/CT scan of abdomen and pelvis were performed prior to and after completion of treatment of each patient.

\section{Chemotherapy:}

The CT schedule is as follows:

Inj cisplatin $50 \mathrm{mg} / \mathrm{m} 2$ on day 1 and 2 in divided doses. The drug was given in an infusion over a period of 90 minute after adequate hydration and antiemetics followed by mannitol diuresis.

5fluorouracil :- It was given in a dose of $500 \mathrm{mg} / \mathrm{m} 2$ over 6 hours on day 1 and day 2 . Chemotherapy was repeated after 3 weeks after checking CHG, LFT, KFT before every cycle of chemotherapy. Two cycles of neoadjuvant chemotherapy was given.

The CT was administered on an out patient basis in a day care room. ECOG toxicity criteria was used for monitoring and documentation of hematological toxicities. The RTOG toxicity criteria was used for radiation induced toxicities ${ }^{[9]}$.

\section{Radiotherapy:}

Radiotherapy (RT) was started within one week of randomization or within 2-3 weeks of completing the second cycle of chemotherapy. RT treatment was same in both arms. External beam radiation therapy was administered using cobalt 60 teletherapy machine.

A dose of 45 Gy in 20 fractions in 4 weeks was given at a dose of 225 centi gray per fraction daily, for 5 days in a week.

After a gap of 1 to 2 weeks patients were reassessed for response and patient with good local response and preserved local anatomy were subjected to intracavitary brachytherapy using Selectron remote controlled LDR system, ${ }^{137} \mathrm{Cs}$ based, giving a dose of $35 \mathrm{~Gy}$ to point $\mathrm{A}$.

Patients with poor response or when local anatomy was not preserved and not fit for intracavitary brachytherapy were given external beam radiation therapy in the form of supplement radiation therapy a dose of 20 Gy in 10 fraction over two weeks.

\section{Assessment:}

All patients in CT-RT group were examined prior to each cycle of CT and prior to RT by the same team to assess the response. Patients on RT were seen every week to assess side effects. RT response was assessed 4 weeks following completion of treatment.

\section{Follow up:}

Patients were followed in an outpatient clinic monthly during first year, every two months during second year and every three months during third year. The patients were finally assessed at 36 months from the start of study.The time was calculated from study entry. Tumor recurrence was defined as the presence of biopsy proven cancer three months after completion of RT. Patients with progressive/ recurrent disease were offered palliative CT or symptomatic treatment.

Statistical analysis: The data collected was analyzed by Statistical Package for Social Sciences (SPSS) package 14. The statistical tests used was chi-square to determine the effect of prognostic factors on response to CT. A level of $P \leq 0.05$ was considered statistically significant and $P \leq 0.001$ was taken as highly statistically 
significant. Survival was defined as the time from study entry to date of death. Disease free survival was defined as the time from completion of RT treatment to the date of recurrence of disease.

\section{(CT-RT Group Study Group)}

\section{Results}

Chemotherapy: Fifty- four of 58 patients randomized to CT-RT group were evaluable for response. Two patients refused further CT and two patients were lost to follow up after first course of CT, but these patients have been included for CT toxicity and survival analysis. Fifty- two of the 54(96.30\%) evaluable patients responded: One patient had cCR( $1.85 \%) 51$ had cPR(94.45\%). and 2(3.70\%)patients progressed(Table 2 ). The stage of the disease, histological grade and history of smoking had no influence on response to CT .However, patients aged $>45 \mathrm{yrs}$ and those with haemoglobin $>10 \mathrm{gm} / \mathrm{dl}$ at diagnosis had a slightly better response rate. 2 patients who progressed were poorly differentiated carcinoma, stage IIIB with B/L parametrial involvement up to pelvic wall, nodulo infiltrative growth(Table 3 ).

Radiotherapy after NACT: 52 of 54 patients completed RT. Two patients did not receive the RT as planned. One patient felt that she was well enough and refused RT, one developed vesicovaginal fistula and RT was deferred. Following RT 38(73.08\%) patients achieved CR. 13(25\%) had residual disease, and one (1.92\%) had progressive disease .

Toxicity to Chemotherapy

A total of 104 CT cycles were delivered. Side effects to CT according to ECOG Criteria are shown in Table[4]. Nausea/ vomiting, were major side effects observed(Table 4).

\section{Radiotherapy alone ( $R$ T Group)}

Fifty- four of 55 patients randomized to RT group were evaluable for response. One patient felt that she was well and did not need RT and did not give consent for RT. Forty-one (75.93\%) achieved CR , 12(22.22\%) had residual disease and one patient (1.85\%) progressed(Table 2). Patients with stage IIB disease had a higher response rate $(22 / 29,75.8 \%)$ compared to patients with IIIB disease $(15 / 26,57.7 \%)$, p-value was NS. The major side effects of RT were local skin reactions and lower GI toxicity(Table 4). There was no significant difference in the toxicity in the toxicity of RT between the two groups.

Pattern of Treatment Failure in Two Groups

In CT-RT group 19 patients had treatment failure: 16 at local site, 1 as distant metastasis and 2 with both local and distant metastasis.In RT alone group, 18 patients failed therapy, with recurrence locally in 14, distant in 3 and 1 both local and distant metastasis. The difference between the two groups(CT-RT vs RT) was not significant.

\section{Survival}

CT-RT group:The survival at 36 months for all patients with stage IIB and IIIB the cumulative survival is $64.15 \%$.Patients with stage IIB had better survival rates compared to IIIB.Patients who responded to $\mathrm{CT}(\mathrm{CR}, \mathrm{PR})$ had slightly better survival than $\mathrm{CT}$ non responders.

RT group: Estimated cumulative survival was $66.66 \%$ for the whole group. There was better survival in stage IIB than stage IIIB disease. The difference in survival between the stage IIB and stage IIIB was not significant.

There was no statistically significant difference in overall survival between the two groups. The estimated disease-free survival in the CT-RT groups is 64.15\% (all stages) and for RT group alone is 66.66\%(all stages). The estimated disease free survival in both groups was better for stage IIB than IIIB but was not statistically significant.

\section{Discussion}

Even though the standard of care at present for locally advanced cervical cancer is concurrent chemoradiation ${ }^{[6,7,10]}$ We conducted two prospective randomized control trials in our institution prior to this trial in 2004 \& 2005, wherein we compared concurrent chemoradiation versus radiation alone in locally advanced carcinoma cervix. In both the studies we did not find any benefit of concurrent chemoradiotherapy over radiation alone in our patients and at the same time patients had increased treatment related toxicities in chemoradiotherapy $\operatorname{arm}^{[8]}$.

Many investigators in the past have used neoadjuvant chemotherapy prior to Radiotherapy or surgery in the treatment of locally advanced cervical cancer with significant responses(30\% to 90\%), including complete resposes in $0-47 \%$ of patients, have been achieved ${ }^{[11-29]}$

Our study confirm these observations. We used a combination of cisplatinum and 5- florouracil ,both act as radiosesitizers also. The achievement of a $96 \%$ overall response rate in previously untreated patients is similar to that reported by Kim et al ${ }^{[14]}$ and Pacini et al ${ }^{[18]}$. These response rates are also comparable with other studies using different chemotherapy regimens. ${ }^{[16-22]}$ 
The clinical complete response (cCR) to chemotherapy (CT) in our study(1.92\%) is low as compared to previous studies ${ }^{[14,18,20,22,23]}$. Possible reasons for the low CR includes the use of only two cycles of CT compared to three or more in other studies ${ }^{[14,18,23]}$ and lower platinum dose used ${ }^{[18,20,22,24]}$. Experience with neoadjuvant CT in similar tumors such as head and neck squamous cell cancer indicates that three courses of cisplatinum and 5-flourouracil result in higher CR rate $(>50 \%)$ as compared to $<20 \%$ with one or two courses .The addition of a third course of CT converted many of the partial responders to $\mathrm{CR}^{[30,31]}$.In our study $>90 \%$ partial responders could have become CR after a third course of CT.

We studied a number of variables as potential predictors of response to CT. Stage of the disease, histological grade, history of smoking and extent of parametrial involvement(stage IIIB) did not influence response to CT. Tumor size and parametrial involvement have been reported to be important predictors of CT response in earlier studies ${ }^{[14,15,19]}$.A haemoglobin level $>10 \mathrm{gm} \%$ was predictive of response to CT .A similar finding was reported by Sardi et ${ }^{\mathrm{al}[15]}$. In addition study ,patients $>45$ years of age responded better.

The chemotherapy was generally well tolerated. There was no evidence that CT enhanced the acute and late side effects of subsequent RT.

Patients who responded to CT had no significantly improved disease free survival compared to non responders. Further, the response to $\mathrm{CT}$ was not predictive of response to RT and vice versa. Also in vitro resistance to RT has been ascribed to the presence of the raf oncogene ${ }^{[23]}$. Whether this finding reflects the mechanism of chemoresistance is not clearly not known.

The overall and disease free survival in the CT-RT and RT groups in the present study was not statistically significantly different. Despite the significant responses to CT in most studies in the past, survival for the patients in the CT arm has not been improved. This finding may be due to the use of relatively inactive chemotherapeutic regimens, lack of sufficient dose intensity, or insufficient length of treatment resulting in an unacceptably low CR rate. From both nonrandomized ${ }^{[11-24]}$ and randomized ${ }^{[25-29]}$ studies it is clear that even at a given stage the patient population is heterogenous(eg, tumor size $<5 \mathrm{~cm}$ vs $>5 \mathrm{~cm}$, bilateral parametrial involvement complete or less than complete, presence or absence of lymph node metastasis), which may result in inherent survival or deficit. Whether the addition of adjuvant (CT or biological) ${ }^{[24]}$ therapy after completion of $\mathrm{CT}$ and RT may result in a survival advantage needs further study.

In conclusion, cisplatinum and 5 flourouracil CT prior to RT results in a high overall response rate, although the CR rate is low. Response to CT does not predict response to RT. There is no increase in the toxicity to subsequent RT. Our study has not proven any significant difference in overall and disease-free survival when neoadjuvant $\mathrm{CT}$ is added to $\mathrm{RT}$ regimen.

Conflict of Intrest: "The author(s) declare that they have no conflict of interest."

Abbreviations:

CT chemotherapy

RT radiotherapy

CT- RT chemotherapy followed by radiotherapy

RT radiotherapy alone

cCR clinical complete response

PR partial response

PD progressive disease

5 -FU 5 flourouracil

\section{References}

[1] Parkin DM, Pisani P, Ferlay J,(1993). Estimates of the worldwide incidence of eighteen major cancers in 1985. Int J Cancer, 19,54, 594-606.

[2] National Cancer Registry Program. Biennial report 1988-89(1992):An epidemiological study,Indian Council of Medical Research New Delhi, India, 23-25.

[3] FIGO staging for cancer cervix uteri(1987).UICC manual for classification of malignant tumors, Springer- Verlag, Berlin

[4] Thar T, Million R and Daly J(1982). Radiation treatment of carcinoma of cervix, semin. Oncol.9, 299-311.

[5] Kumar L and Bhargava L,(1991). Chemotherapy in recurrent and advanced cervical cancer. Gynecol.Oncol, 40,107-111.

[6] NCI Issues Clinical Announcement on Cervical Cancer,Chemotherapy Plus Radiation Improves Survival. http://www.nih.gov/news/pr/feb99/nci-22.htm

[7] Lukka H, Hirte H, Fyles A, et al (2002) Concurrent cisplatin-based chemotherapy plus RT for cervical cancer: A meta-analysis. Clin Oncol (R Coll Radiol) 14:203-212.

[8] Negi RR, Gupta M, Kumar M, Gupta MK, Seam R, Rastogi M,( 2010) Concurrent chemoradiation in locally advanced cervix patients. J Can, Res Ther, 6,159-66.

[9] ECOG, Common toxicity criteria. Available from http://ecog.dfci.harvard.edu/general/ctc.pdf. Last accessed from 2007 Dec 10.

[10] Green J, Kirwan J, Tierney J, et al.(2001) Concomitant chemotherapy and radiation therapy for cancer of the uterine cervix. Cochrane Database, CD002225.

[11] Weiner SA, Alberts S, Surhwit DS and Deatherage DK, (1998). A phase II trial of mitomycin, vincristine, bleomycin and cisplatin (MOBP) as neoadjuvant therapy in high risk cervical cancer .Gynaecol Oncol, 30,1-6. 
[12] Kirsten F, Atkinson K.H., Coppelson J.V.M., Elliott P.M., Green D, Houghton R., Murray J .C ,Russel P.,Solomon H .J.,Friedlander M.,Swanson C.E, and Tattershall MHN,(1987). Combination chemotherapyfollowed by surgery or radiotherapy in patients with locally advanced cervical cancer. Br.J.Obstet. Gynaecol.94,583-588.

[13] Symonds R.P ,Burnett R.A, Habeshew T, Kaye S.B, Snee M.P , and Watson E.R.(1989).The prognostic value of chemotherapy given before radiotherapy in advanced cancer of cervix, Br.J Cancer 59,473-475.

[14] Kim D, Moon H., Kim K.,Hwang Y,,Cho S and Kim S(1989). Two year survival, Preoperative adjuvant chemotherapy in treatment of cervical cancer stage IB and II with bulky tumour,Gynaecol. Oncol.33, 225- 230.

[15] Sardi J, Sananes C, Giaroli A, Maya G , and di-Paola G(1990). Neoadjuvant chemotherapy in locally advanced carcinoma of the cervix uteri, Gynaecol. Oncol.38, 486-493.

[16] Lara P C ,Garcia Puche J L and Pedraza V(1990). Cisplatin- ifosfamide as neoadjuvant chemotherapy in stage IIIB cervical uterine squamous cell carcinoma,Cancer Chemother, Pharmacol.26,36-38.

[17] Kuhnle H , Meerpohl H G ,Eiermann W,Roben S ,Lenaz L , and Achterrath(1990). Phase II study of carboplatin/ifosfamide in untreated cervical cancer, Cancer Chemother.Pharmacol.26,33-35.

[18] Panici PB, Greggi S, Scambia G ,Ragusa G, Battaglia F, Coronetta F, and Mancuso S(1991). High-dose cisplatin and bleomycin neoadjuvant chemotherapy plus radical surgery in locally advanced cervical carcinoma; A preliminary report, Gynaecol Oncol.41,212-216.

[19] Volterrani F,Tana S, Lozza L, Marchini S ,Bianchi F, Stefanon B , and De PaloG(1990). Sequential chemotherapy-radiotherapy in the treatment of advanced cervical cancer, Cancer Lower Female Genital Tract 8, 217-222.

[20] Panici PB, Scambia G, Baiocchi G, Greggi S, Raguasa G, Gallo A, Conte M ,Battalgia F, Laurelli G, Rabitti C, Capelli A, and Manusco S(1991). Neoadjuvant chemotherapy and radical surgery in locally advanced cervical cancer; Prognostic factors for response and survival,Cancer 67, 372-379.

[21] Rabinovich M G , Focaccia G, Ferreyra R ,Elem Y, Leone B A and Vallejo C T(1991).Neoadjuvant chemotherapy for cervical carcinoma,Obstet.Gynaecol.78,685-688.

[22] Maranz A, Litovska S, Etcheverry G, Cechin G, Requejo H, Loiacono R., Negro A,Goldfarb A ,Barg S and Pascon G(1991).Neoadjuvant chemotherapy(NCT),surgery and concomitant chemoradiotherapy for locally advanced squamous cell carcinoma of uterine cervix: Clinical and histopathological evaluation,Proc .Annu. Meet. Am. Soc. Clin. Oncol.10, A619.

[23] Gamucci I, Calabres I, Della Giulia M, Grecco M D, Agano I,Zupi G, Lumbardi A,Mariani L,and Atlante G(1991).Bleomycin+ifosfamide+cisplatin(BIP), An effective neoadjuvant chemotherapy regimen for cervical carcinoma, Proc. Third Int. Cong. Neoadjuvant Chemother.February 6-9,1991,Paris, France, p.66.

[24] Fontanelli R ,Spatti G, Raspagliesi F, Zunino F,and Di-Re F. (1992).A preoperative single course of high dose cisplatin and bleomycin with glutathione protection in bulky stage IB/II carcinoma of cervix, Ann. Oncol. 3, 117-121.

[25] Chauvergne J, Rohart J, Heron F, Fargeot P, Berlie J, David P , George M, Bergonie F, Lambret C, Baclesse C, et al(1988). Randomized phase III trial of neoadjuvant chemotherapy(CT) +radiotherapy(RT) Vs RT in stage IIB, III carcinoma of cervix: A cooperative study of the French Oncology Centres, Proc. Annu.Meet.Am. Soc.Clin. Oncol. 7,A524.

[26] Tobias J, Buxton E J, Blackledge G Mould J J, Monagham J, Spooner D, and Chetiyawardana A(1990).Neoadjuvant bleomycin, ifosfamide and cisplatin in cervical cancer,Cancer Chemother.Pharmacol.26,59-62.

[27] Cardenas J, Olguin A, Figueroa F, and Hulzar R. M.(1991). Randomized neoadjuvant chemotherapy in cervical carcinoma stage IIB, PEC+RT Vs RT, Proc. Annu.Meet. Am.Soc. Clin.Oncol.10, A620.

[28] Souhami L, Gil R. A, Allan S, Canaray P C, Araujo C M, Pinto L.H and Silveria T R.(1991).Randomized trial of chemotherapy followed by pelvic radiotherapy alone in stage IIIB carcinoma of the cervix. J. Clin. Oncol. 9, 970-977.

[29] Lalit K et al,.Chemotherapy followed by radiotherapy versus radiotherapy alone in locally advanced cervical cancer, A randomized study(1994).Gynaecologic Oncology. 54,307-315.

[30] Rooney $\mathrm{M}$ et al(1985). Improved complete response rate and survival in advanced head and neck cancer after three courses of induction chemotherapy with 120 hour 5-flourocil infusion and cisplatin, Cancer.55,1123-28.

[31] Clark J et al.(1988). Chemotherapeutic strategies in multidisciplinary treatment of head and neck cancer.Semin.Oncol.15,35-44.

Table 1. Patient Characteristics

\begin{tabular}{lcc}
\hline Age(years) & CT-RT group & RT group \\
\hline Median & 50 & 50 \\
Range & $25-60$ & $25-60$ \\
FIGO stage & 28 & \\
IIB & 30 & 29 \\
IIIB & 16 & 26 \\
Histology grade & 26 & 12 \\
Well- differentiated & 7 & 30 \\
Moderately differentiated & 9 & 5 \\
Poorly differentiated & & 8 \\
Differentiation unknown & &
\end{tabular}


Table 2. Response to treatment

\begin{tabular}{lc}
\hline CT-RT group & \\
Post chemotherapy $(\mathrm{n}=54)$ & \\
Complete & $1(1.78 \%)$ \\
Partial & $51(94.44 \%)$ \\
No response/ Progressive disease & $2(3.58 \%)$ \\
Response rate & $54(96.42 \%)$ \\
Post radiotherapy $(\mathrm{n}=52)$ & \\
Complete & $38(73.08 \%)$ \\
Residual disease & $13(25.00 \%)$ \\
Progressive disease & $1(1.92 \%)$ \\
RT alone group $(\mathrm{n}=54)$ & \\
Complete & $41(75.93 \%)$ \\
Residual disease & $12(22.22 \%)$ \\
Progressive disease & $1(1.85 \%)$
\end{tabular}

Table 3: CT-RT Group: Chemotherapy response according to clinical variables

\begin{tabular}{|c|c|c|c|c|c|c|}
\hline Variable & $\begin{array}{l}\text { No. of } \\
\text { atients }\end{array}$ & $\mathrm{CR}$ & PR & $\mathrm{CR}+\mathrm{PR}(\%)$ & $\mathrm{NR}+\mathrm{PD}$ & $P$ value \\
\hline \multicolumn{7}{|l|}{ Age (Years) } \\
\hline$<45$ & 18 & - & 16 & $16(88.8)$ & 2 & NS \\
\hline$>45$ & 38 & 1 & 37 & $38(100)$ & - & \\
\hline \multicolumn{7}{|l|}{ History of smoking } \\
\hline Yes & 11 & 1 & 10 & $11(100)$ & - & NS \\
\hline No & 45 & - & 43 & $43((95.5)$ & 2 & \\
\hline \multicolumn{7}{|l|}{ Hemoglobin (g/dl) } \\
\hline$<12-13$ & 30 & - & 28 & $28(93.3)$ & 2 & NS \\
\hline$>12$ & 26 & 1 & 25 & $26(100)$ & - & \\
\hline \multicolumn{7}{|l|}{ FIGO Stage } \\
\hline IIB & 27 & 1 & 26 & $27(100)$ & - & NS \\
\hline IIIB & 29 & - & 27 & $27(93.1)$ & 2 & \\
\hline \multicolumn{7}{|c|}{$\begin{array}{l}\text { Parametrial involvement } \\
\text { (Bulk of Disease) }\end{array}$} \\
\hline Unilateral & 25 & 1 & 24 & $25(100)$ & - & NS \\
\hline Bilateral & 31 & - & 29 & $29(93.5)$ & 2 & \\
\hline \multicolumn{7}{|l|}{ Type of Growth } \\
\hline UPG & 44 & 1 & 43 & $44(100)$ & - & NS \\
\hline NPG & 0 & - & - & & - & \\
\hline NIG & 12 & - & 10 & $10(83.3)$ & 2 & NS \\
\hline \multicolumn{7}{|l|}{ Histology } \\
\hline $\mathrm{Sq}$ cell $\mathrm{Ca}$ & 53 & 1 & 52 & $53(100)$ & - & \\
\hline Adeno $\mathrm{Ca}$ & 1 & - & 1 & $1(100)$ & - & \\
\hline Adeno $\mathrm{Sq} \mathrm{Ca}$ & 2 & - & - & - & 2 & \\
\hline \multicolumn{7}{|l|}{ Histologic grade } \\
\hline Well differentiated & 15 & 1 & 14 & $15(100)$ & - & \\
\hline Mod differentiated & 26 & - & 26 & $26(100)$ & - & \\
\hline Poorly differentiated & 7 & - & 5 & $5(71.4)$ & 2 & \\
\hline Differentiation unknown & 8 & - & 8 & $8(100)$ & - & \\
\hline \multicolumn{7}{|l|}{ KPS score } \\
\hline$<80$ & 19 & - & 17 & $17(89.40)$ & 2 & \\
\hline$>80$ & 37 & 1 & 36 & $37(100)$ & - & \\
\hline
\end{tabular}

Table 4 Toxicity to Chemotherapy and Radiotherapy Toxicity to Chemotherapy

ECOG Criteria ( $\%$ course affected)

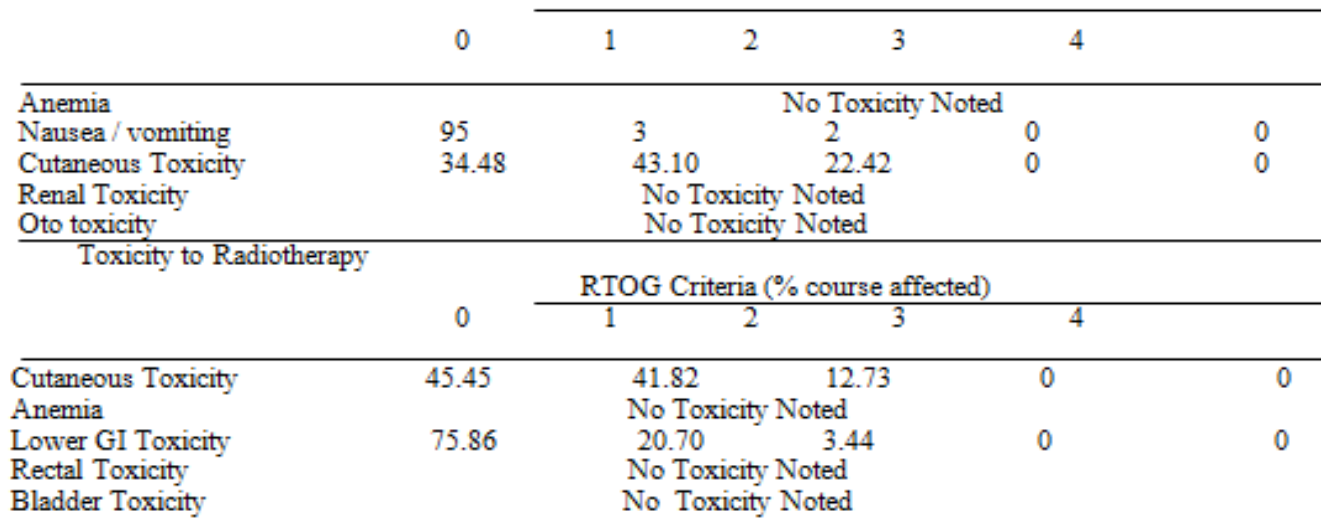

